\title{
Solid Dispersions of Chitosan Glutamate for the Local Delivery of Miconazole: Characterization and In Vitro Activity
}

\author{
R. Pignatello,, ${ }^{*}$ A. Mangiafico ${ }^{2}$, V. Pantò ${ }^{1}$, G. Puglisi ${ }^{1}$ and P.M. Furneri ${ }^{2}$ \\ ${ }^{1}$ Dipartimento di Scienze Farmaceutiche, Università degli Studi di Catania, Città Universitaria, viale A. Doria, 6, \\ I-95125 Catania, Italy \\ ${ }^{2}$ Dipartimento di Scienze Microbiologiche e Scienze Ginecologiche, Università degli Studi di Catania; via Androne, 83, \\ I-95124 Catania, Italy
}

\begin{abstract}
The incorporation of miconazole nitrate (MCN) in mucoadhesive microparticles made of a water-soluble chitosan salt, chitosan glutamate $(\mathrm{CHG})$ was studied. These solid systems may be proposed for buccal or vaginal applications against mycotic infections, taking advantage of the muco-adhesive and permeation enhancing properties of this polymer. Two series of solid dispersions were obtained by either spray-drying and lyophilization, under different formulation variables. The in vitro release of MCN from both the series of microparticles was analyzed at $\mathrm{pH}$ simulating the mouth or vaginal environments. A high amount (60 to $85 \%$ ) of the loaded MCN was released quickly within the first 30 min both at $\mathrm{pH} 5.0$ and 6.6 from all the systems, while the release did not further increase in the following $4 \mathrm{~h}$. These results suggest that the released drug was the fraction of drug desorbed from the CHG particle surface, while a residual amount of drug remained associated or entrapped within them.

An in vitro microbiological assay was carried out against different strains of Candida. MCN- CHG lyophilized solid dispersions gave enhanced inhibitory activity on yeast growth compared to the free drug, whereas the inclusion of $\mathrm{MCN}$ in spray-dried microparticles generally did not improve the activity of the drug. The different physical properties of the two kinds of dispersions seemed to be at the basis of the observed different in vitro antimycotic activity.
\end{abstract}

Keywords: Chitosan glutamate, miconazole, antimycotic activity, microparticles, mucoadhesion, drug delivery system.

\section{INTRODUCTION}

Chitosan $(\mathrm{CH})$ is a hydrophilic, non-toxic biocompatible and biodegradable polysaccharide obtained by alkaline deacetylation of chitin. In the last decades it has been therefore proposed, together with its derivatives and salts, for the production of different drug delivery systems [1-6]. Using $\mathrm{CH}$-based microspheres was recently improved for the preparation of mucoadhesive formulations $[7,8]$. $\mathrm{CH}$ in fact enhances the absorption through the mucosa of small polar molecules, peptides and protein drugs by means of the combination of bioadhesion and transitory accessibility of the tight junctions of cellular membranes [9]. Cell surface and mucin are negatively charged molecules at physiological $\mathrm{pH}$ and therefore good targets for the interaction with positively charged bioadhesive cationic polymers, like $\mathrm{CH}$ [10].

Several types of chitosans are commercially available with different molecular weight, degree of deacetylation, presence of free or substituted acid/amine groups (i.e., chitosan base or chitosan salts) [11]. For their characteristics, chitosans have been proposed as drug carriers for the transmucosal administration in buccal, nasal, ocular, gastrointestinal, and vaginal therapy [12-20].

*Address correspondence to this author at the Dipartimento di Scienze Farmaceutiche, Università degli Studi di Catania; Città Universitaria, viale A. Doria, 6; I-95125 Catania, Italy; Tel: +39 0957384021; Fax: +39 095222239; E-mail: r.pignatello@unict.it
For instance, $\mathrm{CH}$ conjugated to thioglycolic acid proved to be an optimum carrier for vaginal release of antimycotic drugs, such as clotrimazole [21]. Adding $\mathrm{CH}$ to methylcellulose gel preparations allowed to increase the $\mathrm{pH}$ from 2.2 to 4.9 , a value closer to the range of $\mathrm{pH}$ existing in the vaginal habitat [22]. Vaginal tablets formed by $\mathrm{CH}$ and sodium alginate were tested for delivery of metronidazole [23].

Miconazole nitrate (MCN) is an antifungal agent, widely used orally or topically for the treatment of Candida infections of skin [24], mouth [25, 26] and vagina [27]. Such as other antifungal azoles, MCN has been tested under alternative and novel modes of administration [28], including intraocular injections, impregnated bone cement, endobronchial and intrathecal administration; recently, a bioadhesive formulation for the buccal delivery of $\mathrm{MCN}$ has been described [29].

In this work we evaluated the incorporation of $\mathrm{MCN}$ in mucoadhesive microparticles made of a water-soluble $\mathrm{CH}$ salt, chitosan glutamate (CHG). Two series of solid dispersions were obtained by alternative techniques: spray-drying and lyophilization (freeze-drying), using different formulation variables.

These solid systems may be employed for buccal or vaginal applications, in the presence of mycotic affections or local super-infections, taking advantage of the mucoadhesive properties of this polymer. Furthermore, even the mechanism of membrane permeability enhancement is not unquestionably delineated [30], CHG has shown an ability of 
improving the penetration of drugs across mucosae and model cell membranes [31, 32].

The in vitro release of $\mathrm{MCN}$ was studied at different $\mathrm{pH}$ values to evaluate the influence of polymeric matrix swelling properties in simulated buccal or vaginal environments.

The antimycotic activity of the prepared systems was studied by a classic in vitro microbiological assay.

\section{MATERIALS AND METHODS}

\section{Materials}

CHG used in this work (PROTASAN UP G 213) was purchased from FMC Biopolymer (Drammen, Norway). According to the supplier certificate of analysis, viscosity was $131 \mathrm{mPa} \cdot \mathrm{s}, \mathrm{pH} 4.8$, deacetylation degree $85 \%$. The content of glutamic acid was about $43 \%$, that one of proteins about $0.2 \%$.

MCN was kindly provided by Janssen Pharmaceutica N.V. (Beerse, Belgium). Purity was higher than 99.95\% (by HPLC).

\section{Preparation of Drug-Polymer Solid Dispersions by Spray-Drying}

MCN-loaded solid dispersions were prepared using different CHG-to-MCN weight ratios $(2: 1,5: 1,9: 1$, or 19:1). Weighed amounts of polymer and drug (for a constant total amount of $600 \mathrm{mg}$ ) were dispersed under stirring in $100 \mathrm{ml}$ of distilled water; the mixtures were spray-dried with a Mini Büchi B-191 spray-dryer (Büchi Laboratorius-Technik AG, Flawil, Switzerland) using a standard $0.5 \mathrm{~mm}$ nozzle. The process conditions of the spray-drying process were as follows: inlet temperature $104^{\circ} \mathrm{C}$; outlet temperature $45-65^{\circ} \mathrm{C}$; spray-pressure about 2 atm and spray-rate of feed $5 \mathrm{ml} / \mathrm{min}$.

The microparticles were harvested from the apparatus collector as a dry, free-flowing material and were kept under vacuum for $48 \mathrm{~h}$ at $30^{\circ} \mathrm{C}$. Blank microspheres were prepared using the same conditions of drug-loaded particles.

\section{Preparation of Lyophilized Solid Dispersions}

$\mathrm{MCN}$ and $\mathrm{CHG}$, at the same weight ratios used in the previous technique were dispersed in distilled water, if necessary with gentle heating at $40^{\circ} \mathrm{C}$. The mixtures were quickly frozen by immersion in liquid nitrogen and lyophilized for $24 \mathrm{~h}$ with an Edward Modulyo apparatus. White, brittle and homogeneous-looking masses were obtained.

Samples from both the techniques were stored at room temperature in amber glass vials, protected from direct light and heat sources.

\section{Yields of Production}

The yields of production were calculated as the weight percentage of the final product after drying, with respect to the initial total amount of drug and polymer used for the preparations.

\section{Drug Content}

The amount of drug incorporated into each solid system was calculated by dispersing an exactly weighed amount of microparticles (about $5 \mathrm{mg}$ ) in $10 \mathrm{ml}$ of UV-grade methanol, under mechanical stirring (Hematology Mixer, Fisher Scien- tific) for $6 \mathrm{~h}$ at room temperature. The resulting dispersion was filtered through a $0.45 \mu \mathrm{m}$ PTFE membrane filter (Whatman) and read by UV spectrophotometry (Shimadzu UV-1601) at the wavelength of $240 \mathrm{~nm}$. The concentration of MCN in each sample was extrapolated from a calibration curve obtained in the same solvent. Each test was performed in triplicate. The actual drug content was expressed as the percentage of drug entrapped with respect to the amount initially used for the preparations (Table 1).

\section{IR Spectroscopy}

Five milligrams of each sample were mixed with $30 \mathrm{mg}$ of $\mathrm{KBr}$ and compressed at 10 ton by a hydraulic press. The resulting tablets were analyzed in comparison with the following samples: the starting (commercial) polymer material $(\mathrm{CHG})$, a polymer specimen heated at $200^{\circ} \mathrm{C}$ for $30 \mathrm{~min}$, a spray-dried polymer sample (CHG-0), pure MCN, a sample of MCN obtained by spray-drying an aqueous solution of the drug, and a drug-polymer physical mixture $(1: 19, \mathrm{w} / \mathrm{w})$, produced by mixing the solid components in a porcelain mortar for $30 \mathrm{~min}$. The last sample corresponds to the CM1 system in terms of drug-polymer ratio.

Table 1. Percent Drug Loading in CHG Matrixes Obtained by Spray-Drying (CMx) or Lyophilization (CMxL)

\begin{tabular}{|c|c|c|}
\hline $\begin{array}{c}\text { Sample (MCN-CHG } \\
\text { Weight Ratio) }\end{array}$ & $\begin{array}{c}\text { Theoretical \% Drug } \\
\text { Loading }\end{array}$ & $\begin{array}{c}\text { Actual \% Drug } \\
\text { Incorporation }\end{array}$ \\
\hline \hline CM4 (1:2) & 33.33 & $33.60 \pm 0.21$ \\
\hline CM3 (1:5) & 16.66 & $19.61 \pm 0.05$ \\
\hline CM2 (1:9) & 10 & $12.70 \pm 1.10$ \\
\hline CM1 (1:19) & 5 & $6.45 \pm 0.85$ \\
\hline CM4L (1:2) & 33.33 & $29.11 \pm 0.84$ \\
\hline CM3L (1:5) & 16.66 & $17.65 \pm 1.01$ \\
\hline CM2L (1:9) & 10 & $12.68 \pm 0.45$ \\
\hline CM1L (1:19) & 5 & $6.70 \pm 0.06$ \\
\hline
\end{tabular}

${ }^{a}$ Mean of three determinations \pm S.D.

\section{Differential Scanning Calorimetry Analysis (DSC)}

The thermal behavior of blank and drug-loaded microparticles and pure MCN were determined using a Mettler DSC $12 \mathrm{E}$ calorimeter, connected to a Haake D8-G thermocryostat. A sample of indium was used as reference standard to calibrate the temperature and the enthalpy changes. An exactly weighed amount of each sample (about $10 \mathrm{mg}$ ) was placed in an $100-\mu 1$ aluminum crucible. An empty pan was used as reference. Each sample was subjected to a heating scan from 5 to $200^{\circ} \mathrm{C}$, at a heating rate of $10^{\circ} \mathrm{C} / \mathrm{min}$.

The results are given as thermograms that show the dependence on the temperature of heat flux exchanged within the sample during the scan.

\section{In Vitro Drug Release}

To follow the release of MCN from the microspheres, small tablets were formed by mixing $25 \mathrm{mg}$ of each solid dispersion with $75 \mathrm{mg}$ of spray-dried lactose. Several tablets 
of each system were prepared, to study the drug release at different time points.

The ingredients were crushed in a porcelain mortar for 15 min. The mixture was compressed ( 2 tons for $10 \mathrm{~s}$ ) into 8$\mathrm{mm}$ diameter tables by means of an IR steel die. The obtained 100-mg tablets were kept in an exsiccation chamber until use. Control tablets containing $1 \mathrm{mg}$ of pure MCN (to ensure steady-state conditions) were prepared similarly.

Drug release from the above tablets was assessed by means of a dialysis technique, using a QuixSep ${ }^{\circledR}$ Micro Dyalizer (Dasit, Cornaredo, Italy). It consisted of a cylinder, closed at one end, with a diameter of $2 \mathrm{~cm}$, a height of 1.5 $\mathrm{cm}$ and a capacity of $1 \mathrm{ml}$. One tablet and $600 \mu \mathrm{l}$ of the receiving phase $(0.06 \mathrm{M}$ citrate buffer solution, $\mathrm{pH} 5.0$ or 0.05 $\mathrm{M}$ phosphate buffer solution, $\mathrm{pH}$ 6.6; Italian Pharmacopoeia, XI Ed.) were put in the cylinder. A natural cellulose dialysis membrane (Spectrapor, cut-off 3,500 Da) was placed on the open surface of the dialyzer and blocked on the QuixSep ${ }^{\circledR}$ through an elastic ring. The complete system was immersed into a flask containing $40 \mathrm{ml}$ of the same phosphate buffer (external phase) and subjected to magnetic stirring at room temperature. At predetermined times the dialyzer was extracted and the (partially broken) tablet was harvested using an exact volume of UV-grade methanol (typically 2-4 ml). The liquid phase contained in the QuixSep ${ }^{\mathbb{R}}$ (initially formed by $600 \mu 1$ of phosphate buffer) was added of $1.4 \mathrm{ml}$ of methanol. The two solutions were separately filtrated $(0.45$ $\mu \mathrm{m}$ PTFE membrane filter) and analyzed by UV as described above.

The results of the two reading were combined to calculate, by difference, the percentage of drug released in the external phase.

\section{Microbiological Assay}

Some isolates of Candida spp. were studied. They included Candida albicans $(\mathrm{n}=2)$, Candida dubliniensis $(\mathrm{n}=1)$, Candida glabrata $(\mathrm{n}=1)$, Candida humicola $(\mathrm{n}=1)$, Candida krusei $(\mathrm{n}=1)$, Candida parapsilosis $(\mathrm{n}=1)$, and Candida tropicalis $(n=1)$. The isolates were identified to the species level by standard procedures. C. albicans ATCC 90028 reference strain was also included, and C. krusei ATCC 6258 and C. parapsilosis ATCC 22059 were used as quality control strains.

In vitro antifungal susceptibility test was carried out using the NCCLS M27-A2 broth microdilution method [33]. CHG microparticles and pure MCN were diluted (w/v) in RPMI 1640 medium (with L-glutamine and without sodium bicarbonate) (Sigma, Sigma-Aldrich Chimica srl, Milan, Italy) containing $2 \%$ glucose, buffered to a final $\mathrm{pH}$ of 7.0 with $0.165 \mathrm{M}$ MOPS (Sigma). Serial two-fold dilutions (from $64 \mu \mathrm{g} / \mathrm{ml}$ to $0.125 \mu \mathrm{g} / \mathrm{ml}$ ) of each test substance were placed in 96-well microtitre plates.

Inocula were prepared by the spectrophotometic method [33]. The test organisms were grown on Sabouraud Chloramphenicol agar plates (Bio-Mèrieux) at $35^{\circ} \mathrm{C}$ and subcultured at least twice to ensure purity and viability. The inoculum suspension was prepared by picking five colonies, each of at least $1 \mathrm{~mm}$ in diameter, from 24-h-old cultures and suspending the material in $5 \mathrm{ml}$ of sterile $0.85 \% \mathrm{NaCl}$. The turbidity of cell suspensions measured at $530 \mathrm{~nm}$ was adjusted with sterile saline to match the transmittance produced by a $0.5 \mathrm{McFarland}$ barium sulfate standard. This procedure produced a cell suspension containing $1 \times 10^{6}$ to $5 \times 10^{6} \mathrm{CFU} / \mathrm{ml}$ that was then diluted twice with RPMI 1640 medium and distributed among the wells of the microtitre plate $(100$ $\mu 1 /$ well). The final concentration of each inoculum was 0.5 $2.5 \times 10^{3} \mathrm{CFU} / \mathrm{ml}$. The growth control wells contained $100 \mu \mathrm{l}$ of sterile drug-free medium and were inoculated with $100 \mu \mathrm{l}$ of the corresponding inoculum. The quality control organisms were tested in the same manner as the other isolates and were included each time an isolate was tested. In addition, $100 \mu \mathrm{l}$ of uninoculated drug-free medium was included as a sterility control. Plates were sealed and incubated aerobically at $35^{\circ} \mathrm{C}$. The first visual reading was carried out after $24 \mathrm{~h}$ of incubation, and the minimum inhibitory concentration (MIC) value was recorded as the lowest concentration that inhibited visible growth; after $48 \mathrm{~h}$ of incubation, the plates were agitated on a rotating platform to simplify reading of the endpoints, and the lowest concentration that reduced turbidity by $\geq 50 \%$, compared with that in the control-growth well, was recorded as the MIC.

\section{RESULTS AND DISCUSSION}

The two techniques employed, spray-drying and lyophilization, allowed to obtain solid systems of CHG loaded with $\mathrm{MCN}$ at different weight ratios. In the first case, free-flowing microparticles were obtained, while lyophilization produced a light bulk material.

The yield of production was of course quantitative in the case of lyophilization, whereas the spray-drying process allowed to obtain a yield of about $60-65 \%$, well in agreement with other similar studies reported in the literature.

Drug loading values in the prepared solid systems are reported in Table 1. In the solid systems obtained by spraydrying the measured microparticle composition was often variable, indicating that during the preparation the different solubility of the drug and polymer leads to a phase separation and to not homogeneous dispersions.

In a separate experiment (data not shown), weighed amounts of spray-dried microparticles were put in contact under slow stirring with methanol for $15 \mathrm{~min}$ at room temperature. The amount of dissolved MCN was then measured by UV analysis. Considering the limited time of contact with the solvent, the drug dissolved in methanol can be considered to come only from microparticle surface. About one third of the loaded drug was quickly desorbed from the particles in the organic phase: this prompt release indicates a more external localization of MCN in these microparticles, as well as a weak physico-chemical interaction of the drug with the polymeric network.

\section{CHARACTERIZATION OF THE SYSTEMS IN THE SOLID PHASE}

The FT-IR spectrum of CHG showed the characteristic absorption bands at 3436, 2916 e $2850 \mathrm{~cm}^{-1}$, representing respectively the $-\mathrm{OH},-\mathrm{CH}_{2}$ and $-\mathrm{CH}_{3}$ groups (Fig. 1). The amine group gave broad absorption bands between 3400 $3500 \mathrm{~cm}^{-1}$ and around $1670 \mathrm{~cm}^{-1}$. The spectrum of a CHG specimen after spray-drying showed the peak of amino group at $1631 \mathrm{~cm}^{-1}$. After heating at $200^{\circ} \mathrm{C}$, some changes were observed around $1700 \mathrm{~cm}^{-1}$ and especially a shift to higher 
fields of the signal at $1400 \mathrm{~cm}^{-1}$; furthermore, a new signal appeared at $1288 \mathrm{~cm}^{-1}$. All these variations indicate a modification, even though partial, in the chemical structure of the matrix, probably due to a partial deacetylation of chitosan induced by the thermal stress.

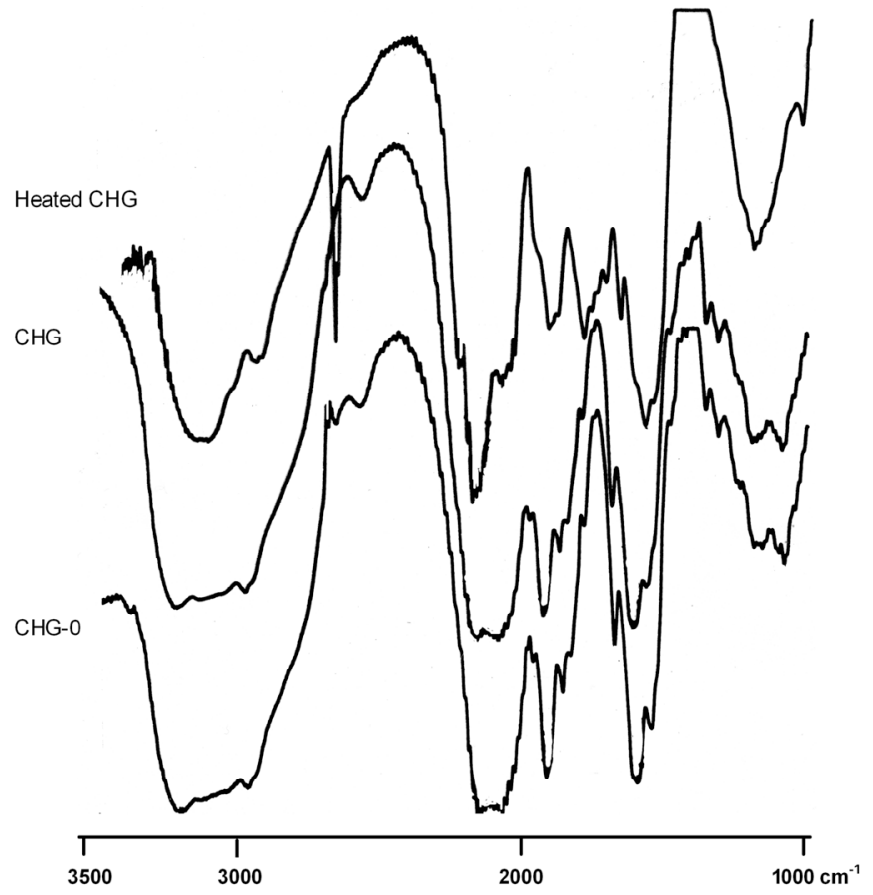

Fig. (1). FT-IR spectra of CHG, a spray-dried CHG sample (CHG0) and a polymer sample heated for $15 \mathrm{~min}$ at $200^{\circ} \mathrm{C}$.

The IR spectrum of MCN displayed three strong peaks between 1700 and $1575 \mathrm{~cm}^{-1}$ (Fig. 2). In the spectra of CHGMCN microparticles prepared by spray-drying the characteristic signals of the drug were masked by the large bands of the polymer network (Fig. (2) reports the spectrum of CM1 as an example). In every case, the drug and the polymer seemed to produce a homogeneous solid solution with low crystallinity, as also confirmed from the DSC experiments (vide infra). Noteworthy, also in the IR spectrum of the physical mixture (Fig. 3), prepared without solvents, the same phenomenon was observed; this suggests that the simple physical mixing of the two ingredients made possible to form a homogeneous mixture.

Finally, the FT-IR spectra of solid systems obtained by lyophilization of the drug and polymer dispersions are reported in Fig. (4). Besides a better resolution of the signals in the region between 1700 and $1500 \mathrm{~cm}^{-1}$, the peaks of the drug after its dispersion in the polymeric matrix were also in this case hardly distinguishable.

On the other hand, the DSC analysis confirmed also the absence of drug crystalline cores in these lyophilized systems, with the exception of the CM4L dispersion, that contains the highest percentage of MCN, and whose DSC run gave a slight endothermic signal at a temperature compatible with that one of MCN fusion ( $c f$. Fig. 8).

The results of DSC calorimetric analysis are given in Figs. (5-8). The thermogram of commercial $\mathrm{CHG}$ showed two endothermic peaks, at $73^{\circ} \mathrm{C}$ and $120^{\circ} \mathrm{C}$, due to the evaporation of adsorbed moisture, and another broad endothermic signal, centred at $175^{\circ} \mathrm{C}$, probably due to the chemi- cal degradation of the material (Fig. 5). The amorphous character of the polymer was confirmed by the absence of clear fusion peaks. The $\mathrm{CHG}$ specimen heated at $200^{\circ} \mathrm{C}$ for $15 \mathrm{~min}$, and then cooled and subjected to the DSC scan, gave an almost void thermogram (Fig. 5), indicating that the material underwent a significant degradation in these conditions, as already remarked in the IR analysis. Moreover, a successive scan of the last sample gave no peak around $175^{\circ} \mathrm{C}$, confirming that the thermal degradation of the polymer was irreversible.

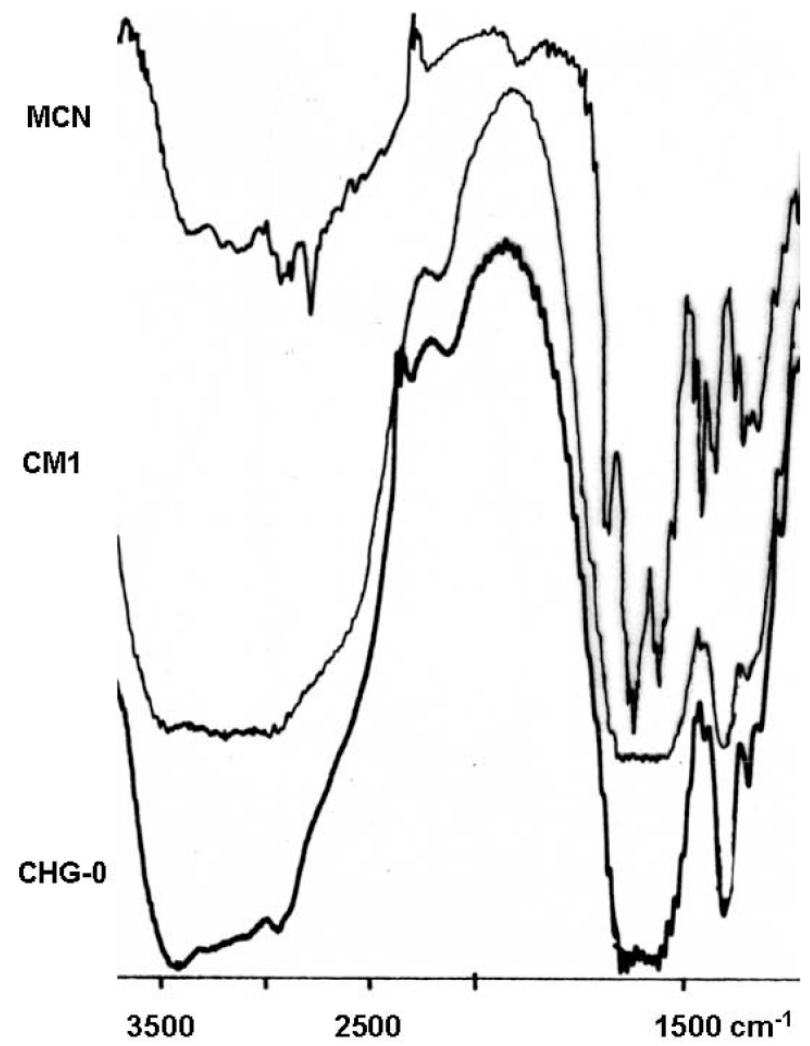

Fig. (2). FT-IR spectra of pure MCN, spray-dried CHG and a microparticle system prepared by spray-drying.

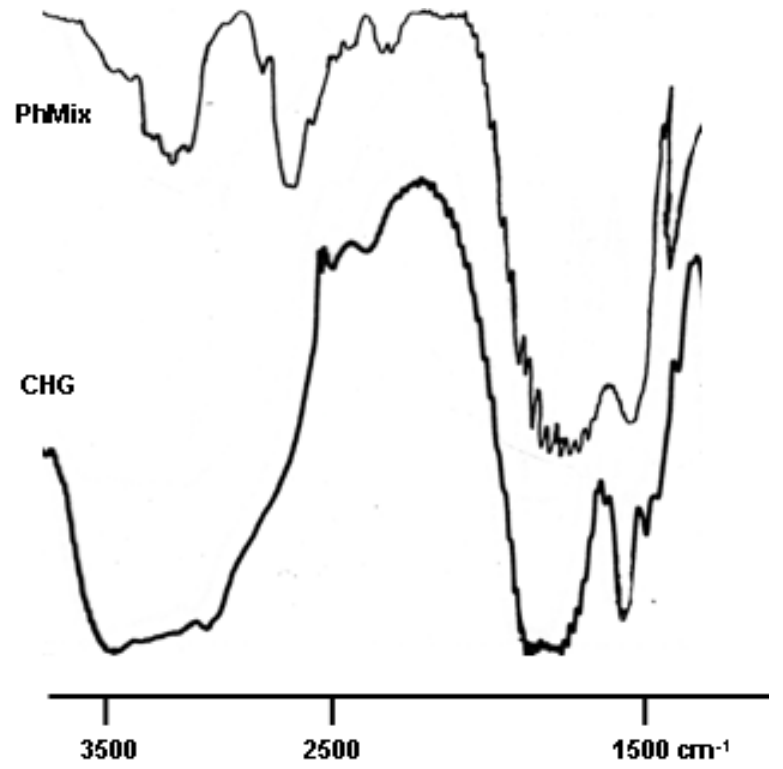

Fig. (3). FT-IR spectra of a CHG-MCN 19:1 (w/w) physical mixture, compared to $\mathrm{CHG}$. 


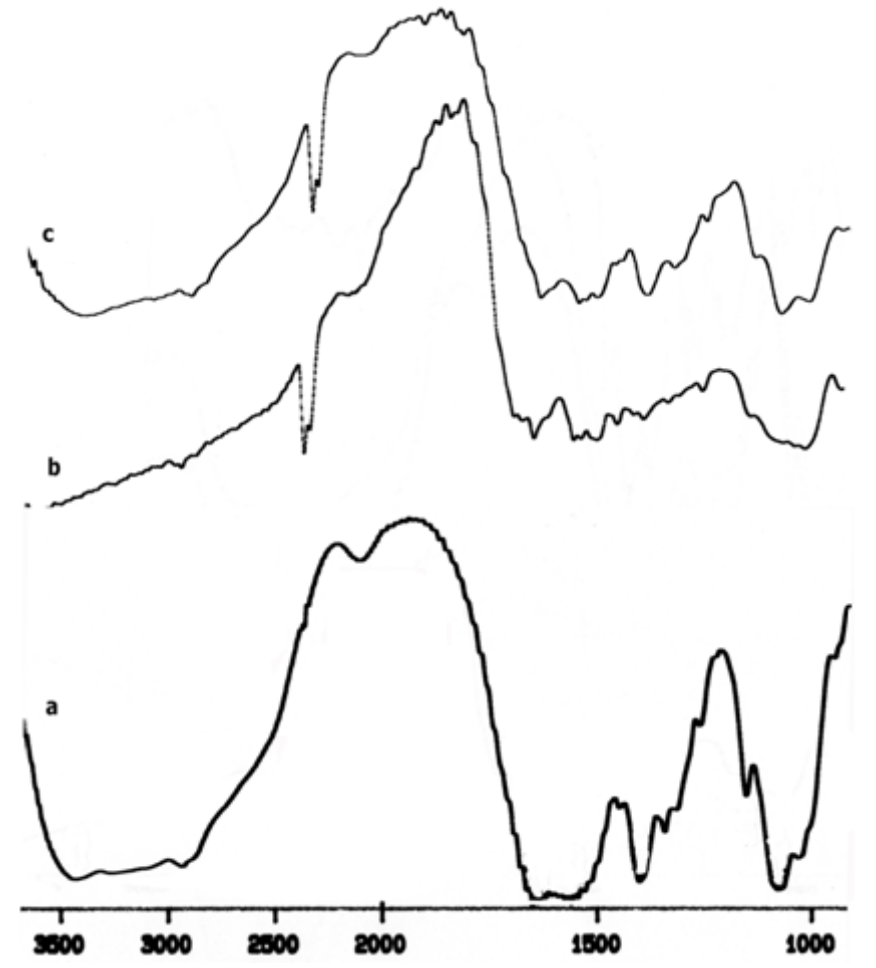

Fig. (4). FT-IR spectra of CHG-MCN microparticle samples prepared by lyophilization: (a) spray-dried CHG-0; (b) CHG-CM3L; (c) CHG-CM4L.

Conversely, the DSC curves of CHG after spray-drying did not show significant differences in comparison with the initial polymer, apart from a trace of residual humidity (Fig. 6). These findings validated the compatibility of the spraydrying operative conditions with such polymer material.

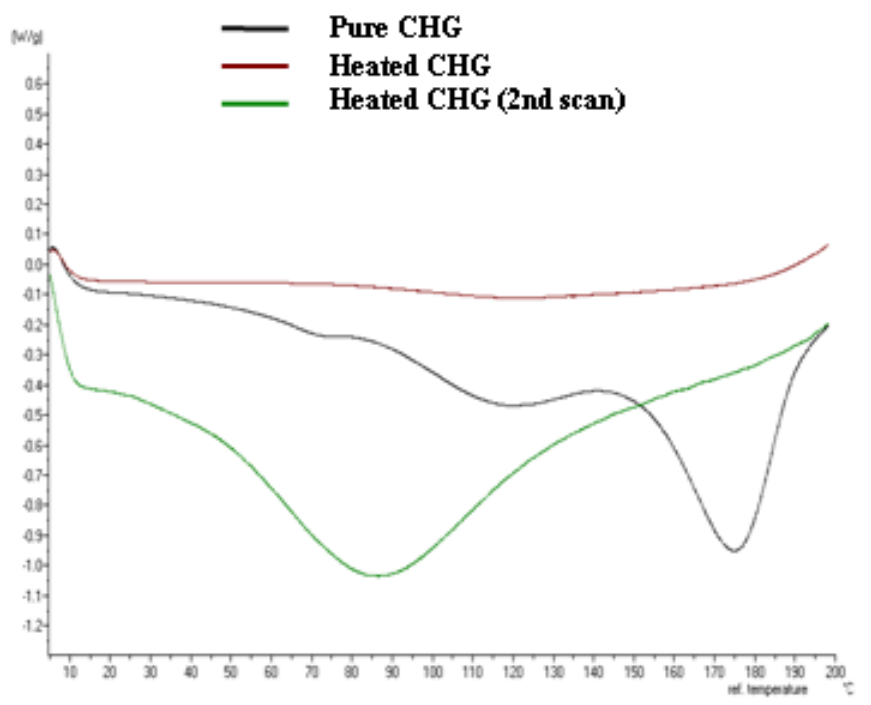

Fig. (5). DSC curve of a commercial CHG sample and after treatment at $200^{\circ} \mathrm{C}$ for $15 \mathrm{~min}$.

Pure MCN showed a clear endothermic peak at $186^{\circ} \mathrm{C}$, corresponding to its fusion temperature (Fig. 6). Such a peak was still present, with the same intensity, also in a drug specimen subjected to the spray-drying (not shown). Thus, the DSC analysis confirmed that the preparative conditions used are compatible with the physico-chemical stability of the drug.
The 19:1 physical mixture gave a DSC profile very similar to that one of pure $\mathrm{CHG}$, with no signal of fusion of MCN crystals (Fig. 6). Such findings can be explained in terms of a complete amorphisation of MCN in the polymer matrix. However, as also suggested by the IR analysis, it is also possible that a chemical interaction between the drug and the polymer occurred through the simple mechanic mixing of the two components in a dry state, leading to the disappearance of free drug crystals.

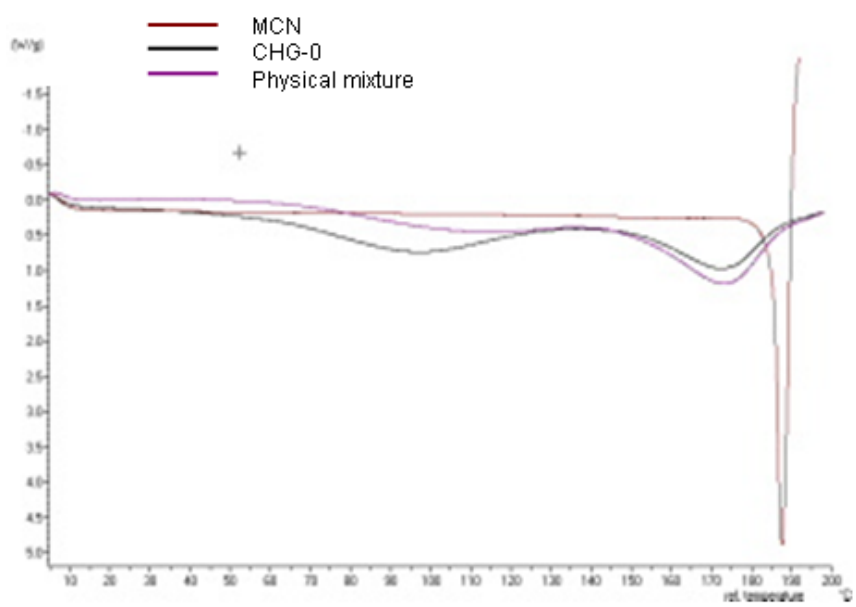

Fig. (6). DSC curves of MCN, a spray-dried CHG sample (CHG-0) and the 19:1 CHG-MCN physical mixture.

The DSC curves of spray-dried microparticles (CM1CM4) and of the freeze-dried samples (CM1L-CM4L) are showed in Figs. (7) and (8), respectively.

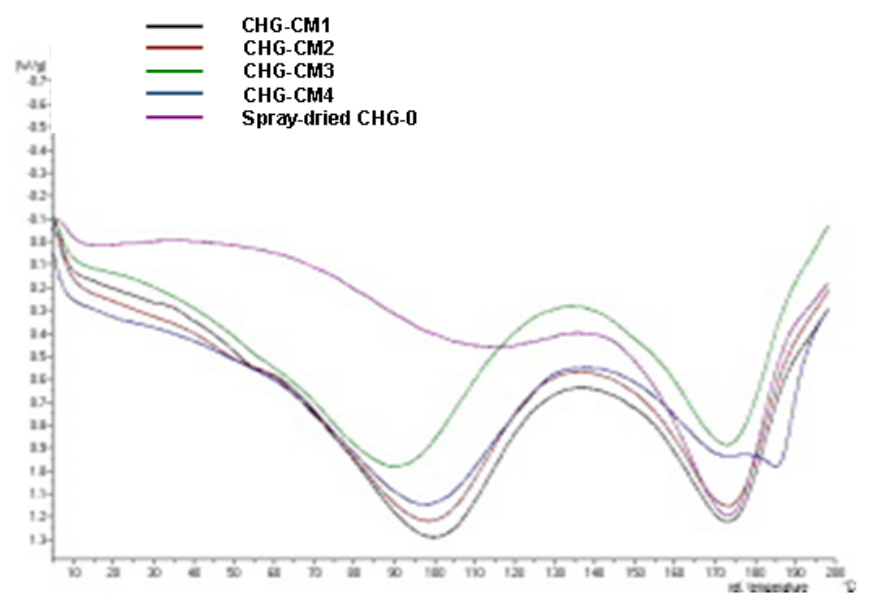

Fig. (7). DSC curves of CHG-MCN microparticles obtained by spray-drying.

The microparticles obtained by spray-drying gave DSC curves very similar to that one of pure CHG (Fig. 7). The release of adsorbed moisture (between $85-110^{\circ} \mathrm{C}$ ), residue of the solvent used in the preparation, was clearly observed, but the large endothermic signal at $175^{\circ} \mathrm{C}$, peculiar of $\mathrm{CHG}$ was still visible. The drug present in these systems did not give any fusion peak, with the exception of the system containing the highest percentage of MCN (CHG-CM4), whose thermogram showed a weakened endothermic signal at a temperature corresponding to MCN fusion.

The DSC results for the prepared solid systems indicated that MCN was dispersed or dissolved homogeneously in the 
polymer matrix in a monomolecular or microcrystalline form, however loosing its original crystalline structure. Over a critical concentration (i.e., a drug-polymer ratio of $1: 2$ ), the solid solution resulting from the two components reached a saturation threshold. The excess drug remained in a crystalline form, as demonstrated by the presence of the corresponding calorimetric fusion signal.

Thus, $\mathrm{CHG}$ and MCN can be considered able to form homogeneous solid dispersions by spray-drying their cosolution; the limit of solubility of the drug in this polymeric matrix ranged between 20\% (CHG-CM3 microparticles) and 33\% (w/w) (CHG-CM4).

The solid systems obtained by co-lyophilization of $\mathrm{CHG}$ and MCN did not show a very different thermotropic behaviour (Fig. 8). Also in these systems the drug appeared to be homogeneously dispersed in the polymeric network, at least up to a $33 \%$ ratio in weight; the system containing a higher drug amount (CHG-CM4L) showed instead a small endothermic peak attributed to the fusion of residual drug crystals (Fig. 8). Furthermore, the large endothermic signal of the polymer was less evident in the latter microparticles, compared to the systems containing lower percentages of drug (CHG-CM1L, CM2L, and CM3L). The polymer was probably completely involved in the physico-chemical interaction with the drug, present in excess with respect to its solubility in the polymeric matrix itself.

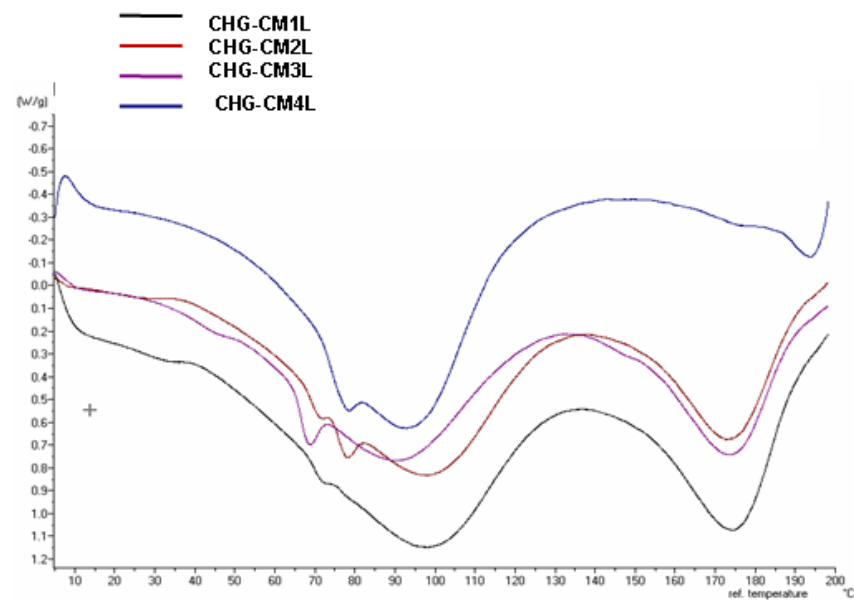

Fig. (8). DSC curves of CHG-MCN solid dispersions obtained by lyophilization.

\section{In Vitro Drug Release}

The release of MCN from spray-dried and lyophilized microparticles was investigated using a receiving aqueous phase buffered at $\mathrm{pH} 6.6$ (the $\mathrm{pH}$ value of saliva). The solid dispersions were converted into tablets before the experiment, to simulate a possible buccal administration form. Moreover, the large volume of receiving phase used in conventional dissolution tests would not give reliable results for a buccal muco-adhesive formulation. Thus, we used a dialysis technique and a device (QuixSep ${ }^{\circledR}$ ) requiring a very small volume of external phase $(<1 \mathrm{ml})$.

$\mathrm{MCN}$ is only very slightly soluble in water. Under the used experimental conditions, only $20 \% \mathrm{MCN}$ went in solution after $2 \mathrm{~h}$, and about $30 \%$ after $6 \mathrm{~h}$ (Fig. 9). On the other hand, chitosan is known to increase the dissolution proper- ties of poorly water soluble drugs [30, 34, 35], due to the reduced size of microparticles and the consequent increased surface area. MCN in fact showed a rapid released from both kinds of solid dispersion, independently on the drug-topolymer ratio (Fig. 9). The percentage of drug released after $30 \mathrm{~min}$ remained then constant for the following $6 \mathrm{~h}$.

By comparing the two techniques of production, interesting differences were observed. In the spray-dried systems, drug release rate was higher and somewhat inversely related to the MCN content in the microparticles, whereas in the lyophilized dispersions a direct relationship between drug loading and its in vitro release rate was observed.

These findings can be explained by considering that in the lyophilized materials lower interactions were originated between the drug and the polymer; thereby, the latter exerts less influence on drug dissolution rate, which was linearly related to MCN concentration in the system (the so-called dissolutive behavior). On the other hands, these systems did not show a real time-dependent drug release profile, but rather a quick desorption from the polymer matrix, as assessed by the above described drug release test in methanol (not shown).

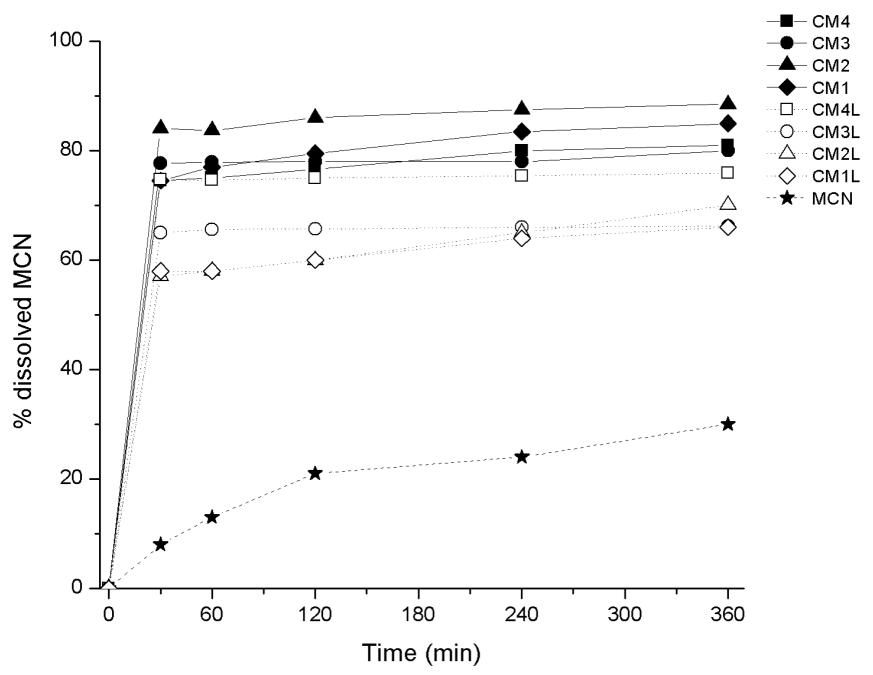

Fig. (9). In vitro $\mathrm{MCN}$ release profile from spray-dried (CMx) or lyophilized $\mathrm{CHG}$ microparticles $(\mathrm{CMxL})$. The receiving phase consisted of a pH 6.6 phosphate buffer solution. Experiments were carried out in triplicate at r.t. Standard deviation (not shown) was within $\pm 4.1 \%$ for all samples.

Conversely, during the spray-drying process a more intimate interaction would occur between the two ingredients. Thus, a higher drug loading would have resulted in a lower permeability of the polymeric network to the diffusion of the dispersed drug. Moreover, the spray-drying process abets the homogeneous dispersion of the drug in the polymeric matrix and the conversion of MCN crystals into microcrystalline or amorphous forms, as suggested by the IR and calorimetry studies. These phenomena could have both enhanced the dissolution rate of the dispersed drug.

The in vitro release test was repeated with the lyophilized microparticles using an aqueous buffer solution at $\mathrm{pH} 5.0$, to simulate a vaginal $\mathrm{pH}$ environment. $\mathrm{MCN}$ has in fact a remarkable therapeutic interest for the treatment of mycotic affections of the genital female apparatus $[36,37]$. 
The $\mathrm{pH}$ of the dissolution buffer markedly affected the release rate of $\mathrm{MCN}$. At $\mathrm{pH} 5$ significant lower amounts of drug were in fact released from the polymer dispersions (Fig. 10), compared to what observed at $\mathrm{pH} 6.6$ (Fig. 9). Except for the CM3L system, also in these $\mathrm{pH}$ conditions a prompt drug release was already observed after $30 \mathrm{~min}$, while the dissolved drug amount remained constant for the following 2 $\mathrm{h}$ of the test.

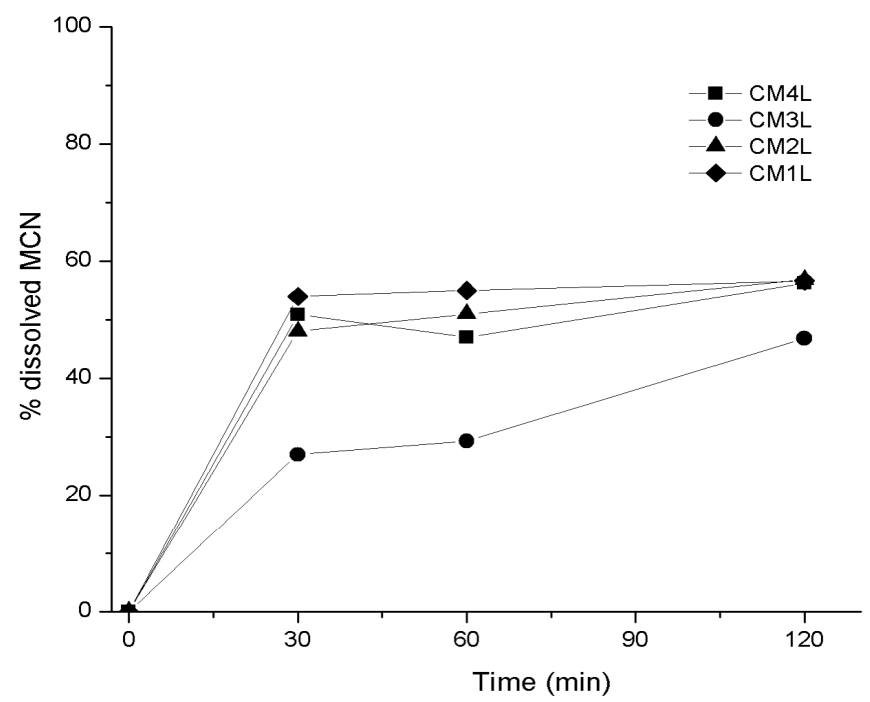

Fig. (10). In vitro $\mathrm{MCN}$ release profile at $\mathrm{pH} 5.0$ from lyophilized CHG microparticles. Experiments were carried out in triplicate at room temperature. Standard deviation (not shown) was within \pm 4.9 $\%$ for all samples.

Chitosans display a higher swelling capacity at acidic $\mathrm{pH}$ values. Therefore, the lower drug release observed at $\mathrm{pH} 5$ can be justified by considering that the lower solubility of $\mathrm{MCN}$ in the buffered aqueous medium was the limiting step that determined the overall drug release rate. In other words, the speed of dissolution of the drug in the two buffer solutions, at $\mathrm{pH} 5$ or 6.6 , determined the amount of drug desorbed from the tested polymeric systems, rather than the ability of the last ones to swell in contact with the aqueous phase and to release the dispersed drug (drug diffusion process).

\section{Microbiological Activity}

Table 2 reports the MIC values measured for pure $\mathrm{MCN}$ or dispersed in the CHG matrixes. As shown, pure CHG did not possess an intrinsic inhibitory activity against the tested Candida strains growth.

Based on the actual MCN concentration in each formulation, the in vitro assay indicated that the antimycotic activity of MCN was enhanced by its dispersion in the polymer (Figs. 11 and 12). In some cases, a 15-20-fold reduction of the MIC values was registered (Fig. 11). The increased activity was particularly remarkable for those Candida strains against which free MCN was less active (e.g., C. Krusei, C. Parapsilosis, C. Tropicalis) (cf. Table 2).

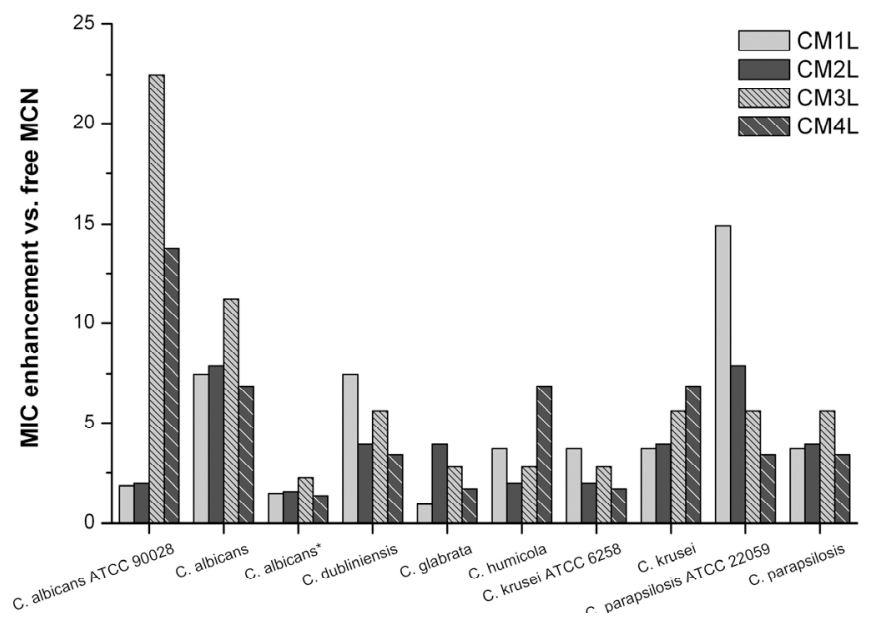

Fig. (11). Enhancement of MIC values after MCN after colyophilization with $\mathrm{CHG}$, in respect to the free drug. Experimental MIC values are given in Table 2.

The lyophilized CHG-MCN dispersions showed a better inhibitory activity on yeast growth than spray-dried microparticles. Although a linear relationship between the actual drug content in the dispersions and the MIC values was observed in general, in many cases a better inhibitory activity against Candida growth was measured for the formulations which contained the highest drug amounts (CM3L and CM4L). Worthy to note, MCN did not undergo any chemi-

Table 2. MIC Values for Pure MCN or Carried in CHG Matrixes. The Activity of Pure CHG is Reported for Comparison. The Actual MCN Concentration in $1 \mathrm{mg}$ of Each Formulation is Reported in Brackets

\begin{tabular}{|c|c|c|c|c|c|c|c|c|c|}
\hline C. albicans ATCC 90028 & 0.5 & 4 & 4 & 2 & 4 & 2 & 0.125 & 0.125 & $>64$ \\
\hline C. albicans & 0.5 & 2 & 2 & 1 & 1 & 0.5 & 0.25 & 0.25 & $>64$ \\
\hline C. albicans* & $<0.125$ & 2 & 2 & 1 & 1 & 0.5 & 0.25 & 0.25 & $>64$ \\
\hline C. dubliniensis & 0.125 & 1 & 0.5 & 0.125 & 0.25 & 0.25 & 0.125 & 0.125 & $>64$ \\
\hline C. humicola & 2 & 4 & 4 & 4 & 8 & 8 & 4 & 1 & 64 \\
\hline C. krusei ATCC 6258 & 4 & 16 & 16 & 16 & 16 & 16 & 8 & 8 & $>64$ \\
\hline C. krusei & 4 & 8 & 8 & 4 & 16 & 8 & 4 & 2 & $>64$ \\
\hline C. parapsilosis ATCC 22059 & 4 & 16 & 16 & 4 & 4 & 4 & 4 & 4 & $>64$ \\
\hline C. parapsilosis & 4 & 32 & 32 & 16 & 16 & 8 & 4 & 4 & $>64$ \\
\hline
\end{tabular}


cal change or degradation under both the preparation processes used, namely spray-drying and freeze-drying, as confirmed by the IR and DSC analysis on pure MCN samples submitted to the same conditions used for the preparation of microspheres. Since also its antimycotic activity should have not been affected by these processes, the measured MIC values would thereby reflect the drug concentration after its release from microspheres in the assay wells. In this respect, a good correlation was obtained between the in vitro release tests (Fig. 9) and the microbiological assay results.

The relatively lower antimycotic activity shown by spray-dried microspheres (Table $\mathbf{2}$ and Fig. (12)) can be ascribed to their physical state. A more compact and dense polymer matrix was obtained through this technique, with respect to the lyophilisation that gave a light material. Consequently, the diffusion of the incorporated $\mathrm{MCN}$ out of the microparticles was less effective and resulted in a lower drug concentration in the culture wells at the time $(48 \mathrm{~h})$ at which the MIC values were registered.

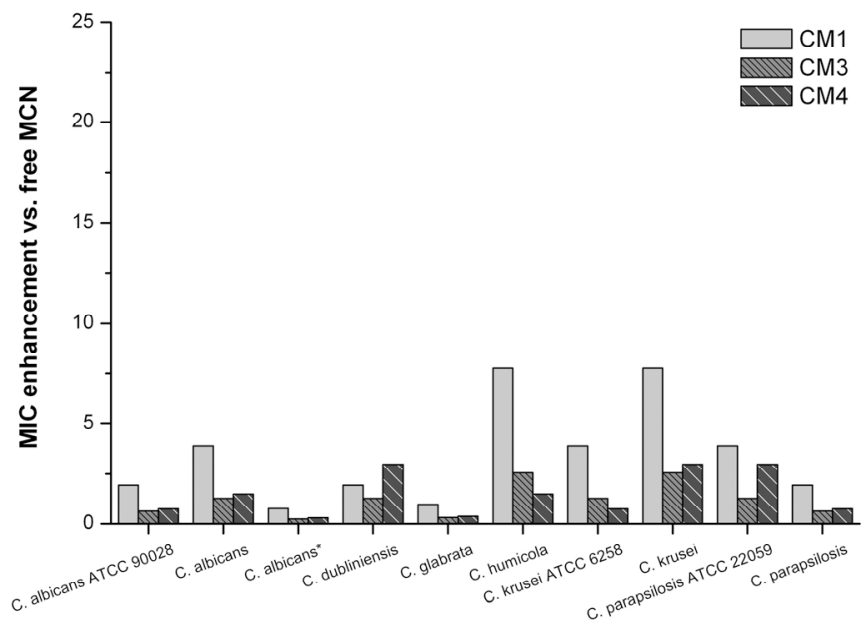

Fig. (12). Enhancement of MIC values for MCN in spray-dried CHG microparticles, with respect to the free drug. Experimental MIC values are given in Table 2.

A final consideration can be made by comparing the in vitro release tests with the microbiological assay: for instance, microparticles CM3 and CM4L gave a very close drug release profile (Fig. 9) but were very different in terms of antimycotic activity (Figs. 11 and 12). Analogously, the lyophilized systems CM1L, CM2L and CM3L, that displayed a similar release profile of the entrapped drug, demonstrated a different in vitro inhibitory activity. These results can of course be originated by the different experimental conditions used, above all the volume of receiving aqueous phase and the presence, in the microbiological assay, of a 'capturing' biophase that drove the diffusion of the drug from the solid particles. However, these considerations suggest to use caution in relating data from the different experiments.

\section{REFERENCES}

[1] Fwu-Long, M.; Tan, Y.-C.; Liang, H.-F.; Sung, H.-W. Biomaterials, 2002, 23, 181.

[2] Fini, A.; Orienti, I. Am. J. Drug Deliv., 2003, 1, 43

[3] Agnihotri, S.A.; Mallikarjuna, N.N.; Aminabhavi, T.M. J. Control. Rel., 2004, 100, 5.

[4] Prego, C.; Torres, D.; Alonso, M.J. Expert Opin. Drug Deliv., 2005, 2,843 .

[5] Jayakumar, R.; New, N.; Tokura, S.; Tamura, H. Int. J. Biol. Macromol., 2006, 40, 175.

[6] Varshosaz, J. Expert Opin. Drug. Deliv., 2007, 4, 263.

[7] Pershing, L.K.; Corlett, J.L.; Nelson, J.L. Pharm. Res., 2002, 19, 270.

[8] Prabaharan, M.; Mano, J.F. Drug Deliv., 2005, 12, 41

[9] Illum, L. Pharm. Res., 2003, 15, 1326.

[10] Woodley, J. Ch. Pharmacokinet., 2001; 40, 77.

[11] Chopra, S.; Mahdi, S.; Kaur, J.; Iqbal, Z.; Talegaonkar, S.; Ahmad, F.J. J. Pharm. Pharmacol., 2006, 58, 1021.

[12] Ceschel, G.C.; Maffei, P.; Lombardi Borgia, S.; Ronchi, C.; Rossi, S. Drug Dev. Ind. Pharm., 2001, 27, 541.

[13] Alonso, M.J.; Sanchez, A. J. Pharm. Pharmacol., 2003, 55, 1451.

[14] Cerchiara, T.; Luppi, B.; Bigucci, F.; Zecchi, V. J. Pharm. Pharmacol., 2003, 55, 1623 .

[15] Hejazi, R.; Amiji, M. J. Control. Rel., 2003, 89, 151.

[16] Cano-Cebrian, M.J.; Zornoza, T.; Granero, L.; Polache, A. Curr. Drug Deliv., 2005, 2, 9.

[17] Valenta, C. Adv. Drug Deliv. Rev., 2005, 57, 1692.

[18] Gavini, E.; Hegge, A.B.; Rassu, G.; Sanna, V.; Testa, C.; Pirisino, G.; Karlsen, J.; Giunchedi, P. Int. J. Pharm., 2006, 307, 9.

[19] Learoyd, T.P.; Burrows, J.L.; French, E.; Seville, P.C. Eur. J. Pharm. Biopharm., 2008, 68, 224

[20] Oosegi, T.; Onishi, H.; Machida, Y. Eur. J. Pharm. Biopharm., 2008, 68, 260

[21] Chang, J.Y.; Oh, Y.K.; Kong, H.S.; Kim, E.J.; Jang, D.D.; Nam, K.T.; Kim, K. J. Control. Rel., 2002, 82, 39.

[22] Mandal, T.K. Eur. J. Pharm. Biopharm., 2000, 50, 337.

[23] El-Kamel, A.; Sokar, M.; Naggar, V.; Al Gamal, S. AAPS Pharm. Sci., 2002, 4, E44.

[24] Minghetti, P.; Cilurzo, F.; Casiraghi, A.; Molla, F.A.; Montanari, L. Drug Dev. Ind. Pharm., 1999, 25, 679.

[25] Bouckaert, S.; Remon, J.P. J. Pharm. Pharmacol., 1993, 45, 504.

[26] Rindum, J.L.; Holmstrup, P.: Pedersen, M.; Rassing, M.R.; Stoltze, K. Scand. J. Dent. Res., 1993, 101, 386.

[27] Fothergill, A.W. Expert Rev. Anti Infect. Ther., 2006, 4, 171.

[28] Arthur, R.R.; Drew, R.H.; Perfect, J.R. Expert Opin. Investig. Drugs, 2004, 13, 903.

[29] Mohammed, F.A.; Khedr, H. Drug Dev. Ind. Pharm., 2003, 29 , 321 .

[30] Maestrelli, F.; Zerrouk, N.; Chemtob, C.; Mura, P. Int. J. Pharm., 2004, 271, 257.

[31] Lim, S.T.; Martin, G.P.; Berry, D.J.; Brown, M.B. J. Control. Rel., 2000, 66, 281.

[32] Portero, A.; Remunain-Lòpez, C.; Nielsen, H.M. Pharm. Res., 2002, 19, 169.

[33] National Committee for Clinical Laboratory Standards. Reference Method for Broth Dilution Antifungal Susceptibility Testing of Yeast: Approved Standard M27-A2. NCCLS, Villanova, PA, USA, 2002.

[34] Portero, A. ; Remunain-Lòpez, C. ; Vila-Jato, J.L. Int. J. Pharm., 1998, 175,75 .

[35] Vijaya Kumar, S.G.; Mishra, D.N. Chem. Pharm. Bull., 2006, 54, 1102 .

[36] Barnhart, K. Curr. Med. Res. Opin., 2005, 21, 127.

[37] Richter, S.S.; Galask, R.P.; Messer, S.A.; Hollis, R.J.; Diekema, D.J.; Pfaller, M.A. J. Clin. Microbiol., 2005, 43, 2155. 